\section{Raman Spectrum and Molecular Structure of Ozone}

AN attempt has been made to find the Raman spectrum of ozone, using a 30 per cent solution of ozone in liquid oxygen and a mercury are as the exciting light. The solution is a dark purple in colour and absorbs the mercury line at $4358 \mathrm{~A}$. quite considerably. The mercury line at $4046 \mathrm{~A}$., however, is scattered fairly strongly, but it has no strong Raman companions. There is an extremely weak doublet corresponding to a mean frequency shift of $1280 \mathrm{~cm}^{-1}$, but no other Raman lines could be observed even with exposures lasting 80 hours. The spectrograph used was not particularly fast, but photographs of the weak oxygen line at $1550 \mathrm{~cm}^{-1}$ from $4046 \mathrm{~A}$. could be obtained in 10 hours.

The conventional representation of the ozone molecule is an arrangement of the oxygen atoms at the corners of an equilateral triangle. More recently a straight line model has been proposed. ${ }^{1}$ The weakness of the Raman spectrum is important as evidence against any simple symmetrical structure of the ozone molecule. On Placzek's theory, ${ }^{2}$ either of these models would give rise to at least one strong Raman line, since each has a symmetrical mode of vibration in which the polarisability varies sharply with changes in the associated normal co-ordinate. The conclusion that the form of the ozone molecule is triangular but not equilateral is supported by the results of investigations of the infra-red absorption bands of gaseous ozone under high dispersion which one of us (S. L. G.) is carrying on at present.

A full account of the latter investigations, along with a discussion of the form of the ozone molecule, will be published later.

G. B. B. M. Sutherland.

S. L. GERHARD.

University of Michigan,

\section{Ann Arbor,}

June 29 .

1 Jakowleva and Kondratjew, Phys. Rev., 39, 533; 1932.

2 Placzek, Z. Physik, 70, 84 ; 1931.

\section{Post-Dissociation Radiation from Sulphur Trioxide}

AcCording to Franck's theory of photodissociation of the halogens, the effect of light absorption is to split $I_{2}$ into a normal iodine $\left({ }^{2} P_{3}\right)$ and an excited iodine $\left({ }^{2} P_{\frac{1}{2}}\right)$. No direct proof has yet been forthcoming that the metastable atoms are actually produced in the reaction.

A direct proof of the production of the ${ }^{2} P_{1}$-atoms may be attempted in various ways. The most convincing proof will be afforded if it can be shown that the illuminated $I_{2}$ vapour gives out radiations of frequency $\nu={ }^{2} P_{3}-{ }^{2} P_{\frac{1}{3}}$ at very low pressures. The low pressure would avoid collisions of the second kind, and thus the energy of excitation would be available again as a radiation. The experiment with $I_{2}$ would be difficult, as the line $v={ }^{2} P_{\frac{3}{3}}-2 P_{\frac{1}{2}}$ would be in the far infra-red. But there is promise of better success with photodissociation of $\mathrm{SO}_{3}$.

It was recently postulated by me ${ }^{1}$ that $\mathrm{SO}_{3}$, on absorption of light of wave-length less than $\lambda 2300 \mathrm{~A}$., decomposes into $\mathrm{SO}_{2}$ and $\mathrm{O}\left({ }^{1} D_{2}\right)$. If the assumptions are correct, it would mean that by irradiating $\mathrm{SO}_{3}$ with light of wave-length below $\lambda 2300$, we would obtain oxygen atoms in the ${ }^{1} D_{2}$-state. If we prevent collisions of the second kind by sufficiently lowering the pressure, the forbidden transition line of the oxygen atom $\mathrm{O}\left({ }^{3} P_{1}-{ }^{1} D_{2}\right)$, corresponding to the wave-length $\lambda 6364$, would be expected from the $\mathrm{SO}_{3}$ gas. ${ }^{2}$

After repeated trials a weak line has been obtained by illuminating for 50 hours a $100 \mathrm{~cm}$. long column of
$\mathrm{SO}_{3}$ gas at a pressure of less than $1 \mathrm{~mm}$. The illuminating source was the condensed spark lines of cadmium below $\lambda 2300$. The wave-length of the observed line is the same as that of the $O\left({ }^{3} P_{1}-{ }^{1} D_{2}\right)$ line, that is, $\lambda 6364$.

Physics Department, Arun. K. Dutta.

University of Allahabad, July 2.

${ }^{1}$ Dutta, "On the Absorption Spectrum of $\mathrm{SO}_{3}$, etc.", communicated to Proc. Roy. Soc.

2 Paschen, Z. Physik, 65, 1; 1930.

\section{A New Photoelectric Phenomenon}

Metal films are subjected to the passage of electric current and exposed to light, which is interrupted with acoustic frequency by a rotating disc with holes. A thermionic amplifier permits a sound of the same frequency to be heard in a telephone when silver, gold, platinum, and tin are tested : aluminium and zinc give no effect.

A copper wheel with sinusoidal border, carrying as many teeth as the holes of the disc, turns jointly with it. Against the border of the wheel, a jet of mercury is directed, the length of which pulsates with the same period as the light. The jet is included in the circuit of the metal film. By regulating the position of the jet and the resistances of the circuit, the sound at the telephone may be extinguished. It may, therefore, be concluded that :

1. The metals examined increase in resistance under action of the light. For silver, the maximum effect seems to be obtained in the ultra-violet region; for the other elements, such a maximum is displaced towards the visible spectrum and perhaps the infra-red.

2. The increase in resistance is of the order of $1 / 10,000$ to $1 / 100,000$ of the resistance of the metal.

The same experiments have been repeated by plunging the metal films into a current of water, but no sensible variation in the intensity of the effect has been noted. The effect appears, therefore, to result from direct action of light on electrical conductivity.

Institute of Physics,

Q. Majorana.

University of Bologna, July 9.

\section{Origin of the Coronal Lines}

IN a recent communication in these columns, Frerichs 1 has criticised de Bruin's classification of the strongest visible lines of the corona. In the same issue of Nature, Dingle ${ }^{2}$ also has criticised the identification of these lines. In each of these communications the authors failed to direct attention to another very strong objection to de Bruin's conclusion that the green auroral line and the green coronal line originate on the same metastable level of oxygen, the ${ }^{1} S_{0}$ level. Since no trace of the green auroral line has ever been observed in the corona, and as no one has ever observed the green coronal line in either the aurora or in laboratory discharges in which the green auroral line was very intense, it is extremely difficult to see how the two lines can originate on the same initial state. If the two lines did originate on the same initial state, their relative intensities should be the same in both the aurora and the corona spectra. One might be able to account for a slight difference in relative intensities by postulating reabsorption of one of the lines, but it seems impossible to explain the profound difference which would follow from de Bruin's classification of the green coronal line.

University of California at Los Angeles, JosEPH KaPLAN. July 2 .

1 NATURE, 129, $901 ; 1932$

2 NATURE, 129, $902 ; 1932$

No. 3276, Vou. 130] 